\title{
Hume sobre los milagros
}

\section{Hume on Miracles}

\author{
Vicente Sanfélix Vidarte ${ }^{1}$ \\ Lidia Tienda Palop ${ }^{2}$ \\ Universidad de Valencia (España)
}

Recibido: 02-03-18

Aprobado: 09-04-18

\section{Resumen}

La tesis sostenida por David Hume en su ensayo sobre los milagros, contenida en el capítulo $\mathrm{X}$ de su primera Investigación, ha sido objeto continuo de interés. Sus detractores han sostenido que el conjunto del argumento de Hume fracasa. Tras reconstruir los dos argumentos proporcionados por Hume -argumento a priori y argumento a posteriori- proponemos establecer una distinción entre milagros ${ }_{\mathrm{n}}$ (acontecimientos naturales extraordinarios)

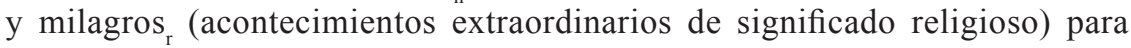
concluir que el argumento de Hume justifica el escepticismo respecto a estos últimos. En definitiva, el argumento de Hume, lejos de pretender en última instancia probar la inexistencia de los milagros como un factum dentro del orden natural, tiene por objeto demarcar la distinción entre creencias científicas y creencias religiosas como dos ámbitos que deben considerarse de manera separada. Esta diferenciación ha contribuido de manera decisiva

\footnotetext{
${ }^{1}$ (Vicente.Sanfelix@uv.es). Catedrático de Filosofía de la Universidad de Valencia. Editor de David Hume. Textos cardinales. Barcelona, Peninsula, 1986, ha cotraducido (junto con Carmen Ors Marqués) la Investigación sobre el entendimiento humano. Madrid, Istmo, 2004 y coordinado, junto con Gerardo López Sastre, el volumen en conmemoración del tercer centenario del nacimiento de David Hume que editó en 2011 la revista Daimon. Así mismo es autor de ensayos referidos al sentido del escepticismo académico de Hume, a sus tesis sobre la identidad personal o sobre nuestra creencia en la existencia del mundo externo.

${ }^{2}$ (Lydia.tienda@uv.es). Ha sido investigadora del Programa del Ministerio de Economía y Competitividad "Juan de la Cierva" en el Departamento de Filosofía de la Universidad de Valencia. Investigadora postdoctoral JSPS en la Universidad de Hokkaido, entre sus publicaciones destacan "El papel de las emociones y la literatura en la deliberación pública", publicado en Arbor, y "La noción plural de sujeto de justicia" en Daimon.
} 
a conformar la separación entre Ciencia y Religión propia de las sociedades secularizadas contemporáneas.

Palabras-clave: Milagros, empirismo, orden natural, teísmo, epistemología.

\section{Abstract}

David Hume's thesis on miracles, sustained in the X chapter of his first Enquiry, has been object of permanet interest. His detractors have maintained that Hume's whole argument fails. After reconstructing the two arguments provided by Hume - a priori argument and a posteriori argument- we propose to set the distinction between miracles $s_{n}$ (extraordinary natural events) and miracles (extraordinary events with religious significance) to conclude that Hume's argument justifies scepticism about the last ones. In short, Hume's argument, far from claiming to prove the nonexistence of miracles as a factum within the natural order ultimately, is intended to demarcate the distinction between scientific beliefs and religious beliefs as two realms that must be considered separately. This differentiation has decisively contributed to shaping the separation between Science and Religion proper to contemporary secularized societies.

Key-words: Miracles, empiricism, natural order, theism, epistemology.

\section{El estado de la cuestión}

En los últimos años los estudiosos de Hume han prestado una atención creciente a su ensayo sobre los milagros (el capítulo X de su primera Enquiry ${ }^{3}$ ). El resultado no ha sido el consenso sobre cómo deba ser interpretado, mucho menos sobre cómo haya de ser evaluado ${ }^{4}$. Nuestro propósito no es zanjar esta discusión -no somos tan ingenuos- sino, mucho más modestamente, mostrar que es posible hacer una lectura caritativa -pero no olvidemos que el principio de caridad es una exigencia metodológica de la honradez hermenéutica- del

\footnotetext{
${ }^{3}$ An Enquiry concerning Human Understanding, que nosotros citaremos por la edición bilingüe inglés-español de Vicente Sanfélix, Investigación sobre el entendimiento humano. Madrid, Istmo, 2004. Citaremos esta obra por la abreviatura EHU, aportando el número de sección y de párrafo. Así, por ejemplo, EHU. 10. 4 significa que el texto al que remitimos se encuentra en el párrafo 4 de la sección 10 de la Investigación. Nos atenemos así a la manera de citar que posibilita la edición crítica de la Enquiry que ha realizado T. Beauchamp para la Clarendon Hume edition series. Oxford. 2010.

4 "Hume's essay on the credibility of miracle reports has always been controversial, with much debate over how it should be interpreted, let alone assessed." Peter Millican, en “Twenty Questions about Hume's 'Of Miracles"' en A. O'Hear (Ed.), Philosophy and Religion. Cambridge University Press, 2011. p 151.

En su edición crítica de la primera Enquiry, Tom Beauchamp enumera hasta 15 respuestas críticas que se dieron al ensayo de Hume entre 1749 y 1774. De ahí que afirme Beauchamp que "The essay on miracles was more extensively discussed than all other sections of EHU combined". T. Beauchamp, "Introduction" to D. Hume, An Enquiry concerning Human Understanding. Oxford, University Press, 2006. p. Lxxxiii.
} 
texto humeano, que permita defenderlo de las críticas más acervas que se le han hecho ${ }^{5}$, y entender el porqué de su innegable impacto en la historia de la filosofía ${ }^{6}$.

\section{La racionalidad de la creencia religiosa}

En nuestra opinión, para hacerse una idea adecuada del específico argumento humeano sobre los milagros es preciso clarificar cuál era su punto de vista general acerca de la racionalidad de la creencia religiosa ${ }^{7}$. En este sentido, nos parece que algunos de sus detractores más radicales ya cometen un tremendo error en el mismo punto de partida de su crítica, cuando atribuyen a Hume alguna clase de teísmo más o menos débil ${ }^{8}$.

${ }^{5}$ Una de ellas es, sin duda, la de John Earman, de cuya ferocidad ya puede darnos una idea el título de la obra que dedicó a exponerla: Hume's Abject Failure. Oxford, University Press, 2000. En la contraportada del libro puede leerse: "Philosophers and historians of ideas have been endlessly fascinated by Hume's famous "Of Miracles", which notoriously argues against the possibility of using eyewitness testimony to establish the credibility of miracles. Hume's admirers declare that he succeeded in offering a line of reasoning that provides "an everlasting check to all kinds of superstitious delusion, and consequently, will be useful as long as the world endures." Even Hume's critics agree that he offered a powerful and ingenious argument that must be answered./ Going against common wisdom, John Earman offers a vital new interpretation of Hume's “Of Miracles". By situating Hume's popular argument in the context of the eighteenth-century debate on miracles, Earman shows Hume's argument to be largely unoriginal and chiefly without merit where it is original."

${ }_{6}^{6}$ Tan innegable que no parece exagerada la afirmación de Don Garrett: "Section X of An Enquiry concerning Human Understanding -entitled 'Of Miracles'- is surely the most famous philosophical treatment of the credibility of reports of miracles ever written". D. Garrett, "Hume on Testimony concerning Miracles" en P. Millikan (Ed.), Reading Hume on Human Understanding. Oxford, Clarendon Press, 2002, p. 301.

${ }^{7}$ Una buena presentación general de la filosofía humeana de la religión sigue siendo la de J.C.A. Gaskin, Hume's Philosophy of Religion. London, MacMillan Press, 1978. En nuestro país, José Luis Tasset hizó una amplia presentación de la misma -que incluye un capítulo dedicado a la cuestión de los milagros- en su edición de D. Hume, Escritos impíos y antirreligiosos. Madrid, Akal, 2005.

8 Es el caso del ya mencionado Earman, quien no duda en afirmar que: "There is some evidence that the youthful Hume struggled against religious feeling. If there was such a struggle, there is not much doubt about how the mature Hume resolved it. If we take Philo's pronouncements in Dialogues Concerning Natural Religion (1776) as a guide, the mature Hume was a theist, albeit of a vague and weak-kneed sort. He seems to have been convinced by the argument from design of the proposition "That the cause or causes of order in the universe probably bear some remote analogy to human intelligence". But he was also convinced that the argument does not permit this undefined intelligence to be given further shape or specificity, and certainly not the specificity that would be needed to support any inference "that affects human life, or can be the source of any action or forbearance." Hume's inconsequential theism was combined with an abhorrence of organized religion, which Hume saw as composed of superstitions that have had almost uniformly baneful effects for mankind. When Cleanthes averred that "Religion, however corrupted, is still better than no religion at all." Philo responded as follows: "How happens it then, if vulgar superstition be so salutory to society, that all history abounds so much with accounts of its pernicious consequences on public affairs? Factions, civil wars, persecutions, subversions of government, oppression, slavery: these are the dismal consequences which always attend its prevalency over the minds of men". J.Earman, Op.cit. pp. 4-5.

Araucaria. Revista Iberoamericana de Filosofia, Politica, Humanidades y Relaciones Internacionales, año 20, ${ }^{\circ} 40$. Segundo semestre de 2018. Pp. 269-283. ISSN 1575-6823 e-ISSN 2340-2199 doi: 10.12795/araucaria.2018.i40.12 
Pues bien, la pregunta que queremos plantear es si por mucho que se debilite el teísmo, incluso hasta degradarlo al nivel del deísmo, puede realmente atribuírsele a Hume. Y el texto clave para responder esta pregunta, suele convenirse, lo constituye la famosa conclusión de Filón en los Dialogues concerning Natural Religion. Veamos lo que allí dejó escrito Hume:

\begin{abstract}
"Si toda la teología natural se reduce, como mantiene mucha gente, a una sola proposición simple, aunque ambigua o por lo menos indefinida: la de que la causa o las causas del orden en el universo presenta probablemente alguna remota analogía con la inteligencia humana; si esta proposición no es susceptible de extensión, de variación o de explicación más concreta, si no proporciona ninguna inferencia que afecte a la vida humana o que pueda ser la fuente de alguna acción o de alguna abstención, y si la analogía, imperfecta como es, no puede extenderse más allá de la inteligencia humana ni puede transferirse, con alguna apariencia de probabilidad, a otras cualidades del espíritu, si tal es realmente el caso, ¿qué otra cosa puede hacer el hombre más inquisitivo, más contemplativo y más religioso sino dar un asentimiento franco y filosófico a la proposición, tantas veces como se presente, y creer que los argumentos sobre los que se apoya superan a las objeciones que se levantan contra ella?"'.
\end{abstract}

Sobre lo que queremos llamar la atención no es sobre la extraordinaria cautela de la conclusión de Filón/Hume ${ }^{10}$-en ella figuran hasta cinco condiciones (cinco “si/if”) - sino, lo que nos parece más importante, sobre la

Cabría preguntarse si este "theism of a vague and weak-kneed sort and inconsequential", que Earman atribuye al maduro Hume, no estaría mejor caracterizado como "deísmo". Desde luego, no cuadra con la definición que algunos autorizados filósofos de la religión dan del teísmo; John Leslie Mackie, por ejemplo, para quien “...is theism, the doctrine that there is a god, and in particular a god as conceived in the central tradition of the main monotheistic religions, including Judaism, Christianity, and Islam." The miracle of theism: Arguments for and against the Existence of God. Oxford, Clarendon Press, 1982, p. 1.

${ }^{9}$ Hemos dado la versión española de Miguel Ángel Quintanilla en D. Hume, Historia natural de la religión. Diálogos sobre la religión natural. Salamanca, Sígueme, 1974, p. 194. El texto original de Hume reza como sigue: "IF the whole of natural theology, as some people seem to maintain, boils down to one simple, though somewhat ambiguous or at least undefined proposition: The cause or causes of order in the universe probably bear some remote analogy to human intelligence; $I F$ this proposition can't be extended, varied, or explained in more detail; $I F$ it yields no inference that affects human life or can be the source of any action or forbearance from acting; and $I F$ the analogy, imperfect as it is, extends only to human intelligence, and can't plausibly be transferred to the other qualities of the mind-IF all this really is the case, what can the most curious, thoughtful, and RELIGIOUS man do except give a plain, philosophical assent to the proposition as often as it comes up, and believe that the arguments on which it is based outweigh the objections against it?". D. Hume, Principal Writings on Religion: including Dialogues Concerning Natural Religion; and The Natural History of Religion / David Hume; edited with an introduction by J. C. A. Gaskin, Oxford: Oxford University Press, 2008 p. 129 (Énfasis - mediante uso de mayúsculas- nuestro).

10 Al hablar de "Filón/Hume" no queremos sugerir que es el escéptico Filón el único personaje que expresa en los Diálogos el punto de vista de Hume. Como es bien sabido, esta es una cuestión muy debatida entre los estudiosos del escocés. Nosotros aceptamos que algunos puntos de vista característicamente humeanos aparecen también en boca del estoico Cleantes. No obstante, pensamos que la conclusión de Filón es la conclusión de Hume.

Araucaria. Revista Iberoamericana de Filosofía, Política, Humanidades y Relaciones Internacionales, año 20, ${ }^{\circ} 40$. Segundo semestre de 2018. Pp. 269-283. ISSN 1575-6823 e-ISSN 2340-2199 doi: 10.12795/araucaria.2018.i40.12 
caracterización que Filón/Hume da de aquel para el que semejante conclusión resultará creíble, a saber: "the most curious, thoughtful, and religious man". La pregunta es obvia: y si esta conclusión fuera propuesta a un hombre "curious and thoughtful" pero no "religious", ¿la aceptaría?

En nuestra opinión, Filón/Hume la hubiera respondido negativamente. Y lo que de ello se sigue es un principio que, según pensamos, caracteriza la orientación general de la filosofía humeana de la religión, a saber: la constatación del orden natural no legitima ninguna conclusión teísta, ni aún cuando este "teísmo" se concibe tan débil que mejor haríamos en 1lamarlo "deísmo". La constatación del orden natural solo puede convertirse en evidencia teísta o deísta, si quien la realiza ya está religiosamente predispuesto.

\section{El argumento de Hume}

Si la constatación del orden natural no equivale a evidencia en favor de ninguna religión natural, salvo para el hombre religioso, ¿qué pasa con el quebrantamiento de aquel orden; esto es: con los milagros? Si alguien testifica haber contemplado uno, ¿puede esta testificación considerarse como una evidencia en favor de alguna particular creencia religiosa? Esta es la cuestión a la que procura responder Hume en la sección X de su primera Enquiry: su famoso "Essay on miracles"

Para establecer su respuesta, en la primera parte del mismo Hume ofrece un argumento -usualmente calificado como a priori, dado que Hume lo apoya sobre el significado que él adscribe a conceptos como el de "fiabilidad de un testimonio", "ley natural", "milagro", etc. - que luego, en la segunda parte, se ve complementado con cuatro consideraciones -usualmente caracterizadas por los estudiosos de la obra humeana como el argumento a posteriori, dado que inciden sobre el carácter que, de facto, tienen los testimonios que atestiguan milagros-. Intentemos reconstruirlo.

\section{Argumento a priori:}

1) Hume parte de un principio que podríamos calificar de ponderación, según el cual el razonador justo siempre adecuará el grado de firmeza de su creencia al de la evidencia en la que se apoya (EHU. 10. 4).

\footnotetext{
11 "Hume's Enquiry was originally published as Philosophical Essays concerning Human Understanding. Hence Section 10, which provoked controversy right from the start, quickly became known as his “essay” on miracles." P. Millican, Op.cit. p. 151 nota 1.
} 
2) A esta premisa Hume añade otra relativa al modo correcto de establecer la credibilidad de un testimonio, según la cual esta es función de dos factores: la fiabilidad del testigo (o de los testigos), por una parte (EHU. 10. 5 y ss), y la probabilidad intrínseca del evento testimoniado, por la otra (EHU: 10. 8).

3) Establecidas estas premisas, pasa Hume a definir un milagro como una "violación de las leyes de la naturaleza" (EHU. 10.12), definición que inmediatamente precisa en una nota: "Un milagro puede ajustadamente definirse como una transgresión de una ley de la naturaleza debida a una particular volición de la Deidad, o a la interposición de algún agente invisible." (EHU. 10.12 n.23).

4) Pues bien, dado que una ley de la naturaleza, para ser tenida por tal, ha de estar respaldada por una experiencia perfectamente uniforme o infalible, o sirviéndonos de la terminología del propio Hume, debe estar probada, lo que de ello se sigue es:

5) que "contra la existencia de cualquier milagro" disponemos de "una prueba completa y directa". (EHU. 10. 12).

Sin embargo, esta no es la última palabra de Hume en la primera parte de su Ensayo, que cierra más bien afirmando la conclusión de que:

6) la credibilidad de un testimonio en favor de un milagro solo sería creíble si la falsedad del mismo fuera más milagrosa que la del hecho reportado; o dicho en otros términos, si la fiabilidad del testigo (o de los testigos) estuviera mejor probada que la ley natural que el milagro testimoniado viola. Pero aun en este caso, advierte Hume, lo que tendríamos sería "una mutua destrucción de argumentos, y el superior solo nos daría una seguridad conforme al grado de fuerza que le resta después de sustraer la del inferior" (EHU.10.13).

Este, en definitiva, es a nuestro parecer el esqueleto del conocido como "argumento a priori", que Hume elabora en la primera parte de su Ensayo sobre los milagros; argumento que inmediatamente complementa en la segunda parte con cuatro consideraciones que, en conjunto, constituyen el así llamado "argumento a posteriori":

a) la deficiente fiabilidad de los testigos de cualquier milagro históricamente atestiguado (EHU. 10. 15);

b) la credulidad que la pasión por lo sorprendente y asombroso provoca en los seres humanos (EHU. 10. 16 y ss.);

c) el hecho de que los relatos sobrenaturales y milagrosos abunden especialmente entre naciones bárbaras e ignorantes (EHU. 10. 20 y ss). Y, por último,

d) el descrédito que los testimonios de milagros aducidos desde religiones contrarias entre sí provoca sobre todos ellos (EHU. 10. 24 y ss.). Consideraciones a partir de las cuales concluye Hume: 
e) "que ningún testimonio a favor de ninguna clase de milagro ha equivalido jamás a una probabilidad, mucho menos a una prueba; y que, incluso suponiendo que hubiera equivalido a una prueba, se le opondría otra prueba, derivada de la misma naturaleza del hecho que se pretende establecer... por consiguiente podemos establecer como máxima que ningún testimonio humano puede tener tanta fuerza como para probar un milagro, y convertirlo en un fundamento justo de cualquier tal sistema de religión." (EHU. 10. 35)

\section{Caridad interpretativa}

La argumentación humeana presenta problemas exegéticos que deben ser resueltos antes de que se la pueda someter a evaluación crítica. Quizás el más evidente es el del alcance de la conclusión que Hume quiere establecer con su argumento. De la literalidad de lo que Hume dice se siguen tres posibles interpretaciones:

1) Hume persigue probar una tesis ontológica: no hay milagros ${ }^{12}$ (véase la premisa 5 del argumento a priori).

2) $\mathrm{O}$ una tesis epistemológica ${ }^{13}$ más moderada que se limita a defender que el grado de credibilidad que podemos otorgar a la testificación favorable al acaecimiento de un milagro es muy bajo ${ }^{14}$ (esta parece ser la conclusión sugerida por el punto 6 del argumento a priori).

3) una tesis epistemológica más radical según la cual no podemos depositar en absoluto ninguna credibilidad en este tipo de testimonios, de modo que cualquier razonador justo los tomará como "materia más apropiada de irrisión que de argumento"15 (EHU. 10.26. La tesis que parece confirmar el texto de EHU. 10. 35 que citamos en el apartado e de nuestra reconstrucción de la parte a posteriori del argumento humeano).

\footnotetext{
12 Esta es, por ejemplo, la interpretación que asumía F. Beckwith en su estudio David Hume's Argument against the Miracles. A Critical Analysis. New York, University Press of America, 1989. También la de R.J. Fogelin, "What Hume Actually Said About Miracles". Hume Studies. vol.16, n.1, 1990.

${ }^{13}$ Las interpretaciones epistemológicas del alcance del argumento de Hume son las más habituales. D. Coleman las bautizó como la "traditional interpretation". "Hume, Miracles, and Lotteries". Hume Studies. vol. 14, n. 2, 1988.

14 A. Flew defendió este punto de vista en el capítulo VIII de su clásica monografía Hume’s Philosophy of Belief. London, Routledge \& Kegan Paul, 1961. Igualmente es la interpretación de J.H. Sobel, "On the Evidence of Testimony for Miracles: A Bayesian Reconstruction of David Hume's Analysis”. Philosophical Quarterly, vol. 37, n. 147, 1987.

${ }^{15} \mathrm{La}$ interpretación de autores con T. Penelhum en su monografía sobre Hume. New York, St. Martin's Press, 1975 o F. Wilson, "The Logic of Probabilities in Hume's Argument against Miracles". Hume Studies. Vol. 15, n. 2, 1989. Para todavía más posibles interpretaciones del alcance de la conclusión de Hume, Cf. el artículo ya citado de Don Garrett, Op. cit. pp. 302 y 303.
} 
Ahora bien, ¿son realmente incompatibles estas conclusiones o admiten una síntesis conciliadora? Probemos. Lo que Hume podría querer establecer es que 1) considerado el asunto de una manera apriorística, el testimonio favorable al acaecimiento de un milagro no podría gozar sino de un grado de credibilidad muy bajo; pero 2) considerando a posteriori el tipo de testigos y las circunstancias de los testimonios que atestiguan el acaecimiento de milagros, su verosimilitud se reduce a cero y, por lo tanto, 3 ) este tipo de testimonio no disminuye lo más mínimo la credibilidad en la vigencia de las leyes naturales, de modo que estas constituyen por sí solas "una prueba completa y directa... contra la existencia de cualquier milagro" EHU.10.12. Consideramos que esta interpretación onto-epistemológica de la conclusión del conjunto del argumento humeano (de su parte a priori más su parte a posteriori) es la más adecuada. Pero todavía hay que precisarla más.

Como ya se apuntó (ved la premisa 3 del argumento a priori), Hume da dos definiciones de "milagro": una más amplia -una violación de las leyes naturalesy una más estricta -una transgresión de las leyes naturales provocada por una particular voluntad de la divinidad-. Por otra parte, Hume no solo habla de "milagros" en su Ensayo sino también de "lo extraordinario y lo maravilloso" (EHU. 10. 8), de "prodigios" (EHU. 10. 24), etc., sin que termine de ser consistente en su uso de esta terminología, pues mientras muchas veces emplea estos términos como sinónimos, en otras ocasiones los contrapone (como, por ejemplo, en EHU. 10. 11: "vamos a suponer que el hecho que afirman, en lugar de ser solo maravilloso, sea realmente milagroso").

A fin de facilitar nuestra tarea interpretativa proponemos aplicar cierta caridad interpretativa, y mejorar la consistencia terminológica del propio Hume,

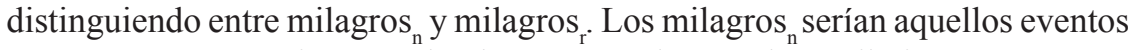
que aunque no se ajustan a las leyes naturales, no les atribuimos una causa sobrenatural o divina. Los milagros, ${ }_{\mathrm{r}}$, por contra, son aquellos acontecimientos a los que atribuimos una causa sobrenatural o divina, un significado religioso.

Pues bien, una vez hecha esta distinción que, reconocemos, no encontraremos literalmente hecha en Hume, pero que, no obstante, creemos que se le puede atribuir razonablemente basándonos en el propio texto de su Ensayo, la pregunta es: la conclusión onto-epistemológica que previamente le atribuimos ¿alcanzaría a ambos tipos de milagros o solo a uno de ellos? Pregunta que no tiene una respuesta fácil porque en el mismo párrafo Hume extiende su conclusión a cualquier clase de milagro, a la vez que parece restringirla a aquellos de los que se quiere hacer "un fundamento justo de cualquier tal sistema de religión" (Véase el ya citado EHU. 10. 35). O dicho en la terminología que recién hemos propuesto: en el mismo párrafo parece defender que su conclusión escéptica es igualmente válida para los milagros ${ }_{n}$ y los milagros ${ }_{\mathrm{r}}$ y restringir su alcance solo a estos últimos. ¿Con cuál de estas dos posibles interpretaciones debemos quedarnos? 
Dado que, justo a continuación del párrafo en que tal notoria ambigüedad se da, precisa Hume lo siguiente: "Pido que las limitaciones aquí señaladas se tengan en cuenta cuando digo que un milagro nunca puede probarse como para resultar el fundamento de un sistema de religión. Pues concedo que, por otra parte, podría haber milagros, o violaciones del curso usual de la naturaleza, de tal clase como para poder probarse por el testimonio humano..." (EHU. 10. 36), para nosotros está claro que la interpretación más adecuada del alcance de la conclusión humeana se restringe a los milagros de los que se quiere hacer fundamento de una particular creencia religiosa. $\mathrm{O}$ dicho con la terminología cuya adopción hemos propuesto: la conclusión escéptica de Hume solo alcanza en su integridad a los milagros. Son estos los que ningún testimonio podría dotar de credibilidad, y de los que la mera existencia de leyes naturales constituye una prueba directa y completa de su inexistencia.

Así precisada nuestra interpretación de las tesis humeanas y de su alcance, una interpretación que consideramos suficientemente razonable a pesar de la falta de precisión terminológica de la que adolece el Ensayo humeano, pensamos que podemos pasar a evaluarlas críticamente.

\section{Crítica}

Nuestra pregunta ahora es si las premisas del argumento humeano son admisibles, y si en su conjunto permiten inferir la conclusión que le hemos atribuido.

Para no demorarnos demasiado preguntémonos por la validez de las dos primeras premisas de la parte a priori del argumento. ¿Es cierto que el "razonador justo" siempre adecúa la firmeza de sus creencias a la de la evidencia sobre las que se apoya; o que la credibilidad que otorgamos a un testimonio está en función de la fiabilidad del informante y de la probabilidad intrínseca del evento testimoniado?

Si la primera premisa de Hume fuera el caso, habría que decir que no hay sobre la faz de la tierra ningún "razonador justo", pues todos estamos absolutamente ciertos de muchas creencias para las que no podemos aducir ninguna evidencia clara más allá, en todo caso, de nuestra confianza en un testimonio difuso. ¿Por qué, por ejemplo, estamos seguros de que existe un país llamado "Islandia", si jamás hemos estado allí y ni siquiera hemos conocido personalmente a nadie que se dijera islandés?

Y esta consideración ya demuestra que la segunda premisa del argumento humeano no es más aceptable que la primera, pues la mayor parte de las veces nos fiamos de testimonios sin tener experiencia de la fiabilidad de quienes nos los proporcionan e, incluso, a pesar de la poca probabilidad intrínseca del hecho 
del que nos informa. Así lo hacemos cuando, por ejemplo, le preguntamos a un desconocido la hora o, por poner un ejemplo clásico, el número de lotería que ha salido ganador en el sorteo recién realizado.

Por otra parte, aunque a pesar de su vacilación quizás pudiéramos dar por buena la definición humeana de "milagro" -el Cambridge Dictionary da como primera acepción del término "an unusual and mysterious event that is thought to have been caused by a god because it does not follow the usual laws of nature"- la premisa cuatro vuelve a resultar muy discutible. ¿Serían compatibles las generalizaciones probabilísticas, que hoy se admiten en la mayor parte de las ciencias, con la "experiencia uniforme e infalible" que Hume reclama como base de las leyes naturales? Y, sobre todo, ¿es cierto que nuestra creencia en estas leyes se fundamenta en una experiencia de ese tipo?

A poco que reflexionemos nos percatamos inmediatamente de que la vasta mayoría de las leyes naturales y verdades generales $-\mathrm{y}$ son estas últimas las que Hume pone como ejemplo, más que las primeras- que damos por válidas, y que forman el fondo de lo que podríamos llamar nuestra imagen del mundo, no las hemos aprendido por experiencia directa, ni las hemos sometido seriamente a prueba. Más bien se nos han transmitido por el mismo testimonio difuso (de nuestros padres, profesores, libros, etc.) al que ya hacíamos alusión cuando comentábamos la primera premisa del argumento humeano ${ }^{16}$.

Todas estas dificultades pueden achacarse a la epistemología rudamente empirista que Hume parece suscribir, pero aparte de ellas todavía hay en la parte a priori de su argumento otras dificultades que podríamos caracterizar como de carácter interno.

Definido un milagro como una violación de una ley natural y una prueba como aquella creencia asentada en una experiencia uniforme e infalible, ¿tiene sentido hablar, como hace Hume en su argumento, de eventos más o menos milagrosos o de pruebas más o menos fuertes? Y suponiendo que consiguiéramos dotar de sentido a esta gradación de lo milagroso o de lo probado -por ejemplo, asumiendo, a la manera de Galileo ${ }^{17}$, que es más milagroso aquel evento que viola más leyes naturales, o que está más probada aquella creencia que tiene a su base una experiencia infalible y uniforme más extensa- ¿es compatible con el empirismo humeano pensar que una cuestión de hecho, como a la postre es que se dé o no determinado tipo de evento, puede zanjarse a priori?

${ }_{16}$ Este punto fue en nuestra opinión claramente establecido por Ludwig Wittgenstein en sus observaciones Sobre la certeza. Barcelona, Gedisa, 1998; y previamente por José Ortega y Gasset en su ensayo sobre "Ideas y creencias", en Obras completas, vol. V, Taurus/Fundación Ortega, Madrid, 2012.

17 Véase el argumento que esgrime el filósofo de Pisa a favor de la interpretación copernicana del milagro de Josué en la carta que dirige a Benedetto Castelli, recogida en Cartas copernicanas. Madrid, Ediciones Alhambra, 1986. 
Del mismo modo, las consideraciones que componen la parte a posteriori del argumento humeano parecen dogmáticas. Aun concediendo a Hume que hasta ahora en todos los casos de testimonios de eventos milagrosos los testigos han resultado poco fiables y las circunstancias sospechosas, e incluso que se han utilizado para minar la credibilidad de otras religiones en cuyo favor también se habían testimoniado hechos milagrosos, ¿tendría que ser necesariamente así? ¿No resulta concebible un testimonio perfectamente respetable, en circunstancias libres de toda sospecha, y realizado en favor de una religión ecuménica, en lugar de excluyente?

Todas estas consideracione ${ }^{18}$ pueden llevarnos a concluir que el argumento de Hume es un completo fiasco. Que sus premisas son materialmente inválidas y muy probablemente hasta inconsistentes con algunos aspectos de la propia epistemología humeana y, por lo tanto, que no sirven para justificar la conclusión que Hume pretende, y que esta es sostenida por el pensador escocés de un modo absolutamente arbitrario y dogmático ${ }^{19}$.

\section{Vindicación de Hume}

Sin embargo, pensamos que el argumento de Hume puede defenderse como válido si se entiende no como un argumento general sobre la epistemología del testimonio, sino como un argumento específico sobre la fiabilidad del testimonio de acontecimientos milagrosos. Veamos.

Es cierto que en circunstancias normales las dos primeras premisas de la parte a priori del argumento son erróneas. Pero no creemos que sea el caso cuando lo testimoniado rompe con, por así decirlo, nuestro "background" cognitivo. Si preguntamos la hora a un desconocido y nos dice las 13'30, confiamos en la veracidad de la información sin pararnos a considerar la probabilidad -escasa- de que efectivamente sean las 13'30... a menos, claro está, que sea noche cerrada. En este caso parece que el análisis humeano de la fiabilidad del testimonio resulta más adecuado: ajustamos el grado de firmeza de nuestra creencia a la evidencia de la que disponemos -premisa 1y sopesamos la probabilidad de que el testimonio sea fiable con la de que el evento testimoniado sea efectivamente el caso - premisa $2-$.

\footnotetext{
18 Estas dificultades han sido reiteradamente señaladas por los estudiosos del ensayo humeano. Para no añadir innecesariamente bibliografía remitimos aquí a los trabajos, ya citados, de P. Millican y de Don Garrett.

19 Entre quienes han considerado el argumento de Hume inválido por cualquiera de estas razones podemos citar a R. Swinburne, The concept of Miracle. London, Palgrave MacMillan, 1970; M.P. Levine, Hume and the Problem of Miracles: A Solution. Dordrecht, Kluwer Academic Publishers, 1989; K. E. Yandell, Hume's 'Inexplicable Mystery'. His Views on Religion. Philadelphia, Temple Press, 1990 (especialmente, la conclusión del capítulo XV); o el ya citado F. Beckwith, op. cit., 1989. 
En cuanto a la premisa 3, la definición de un milagro como un evento que contradice una ley natural, ya la dimos por válida.

La cuestión decisiva es, pues, si podemos dar por válida la premisa 4. ¿Podemos considerar que la admisión de leyes naturales nos compromete con la exclusión de eventos que las contradigan y, por la tanto, la credibilidad de cualquier testimonio que los testifique? ¿No resulta esta tesis absolutamente dogmática e, incluso, contradictoria con el corazón del escepticismo inductivo humeano?

A nuestro entender podemos sacar ahora rentabilidad de nuestra anterior caridad interpretativa, cuando haciendo más coherente la terminología humeana ${\text { distinguimos entre } \text { milagros }_{n} \text { y milagros }}_{r}$, para salvar el argumento humeano. Lo que este nos vendría a decir es que nada impide que un testimonio resulte creíble

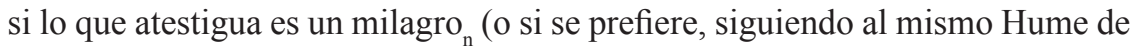
la History of England ${ }^{20}$, si lo que atestigua es algo "extraordinario" -el eclipse de ochos días de duración, que pone como ejemplo hipotético en la segunda parte de la sección $\mathrm{X}_{-}$, siempre y cuando el testimonio cumpla los requisitos de fiabilidad de los testigos y de las circunstancias en que se produjo; pero, por contra, es absolutamente inaceptable cuando lo que atestigua es un milagro ${ }_{r}$ -un milagro en el sentido estricto de la History of England - (a lo que hay que añadir que para Hume habría un caso intermedio entre lo extraordinario y lo milagroso propiamente dicho, a saber: lo "maravilloso" -muy probablemente, en esta categoría debiera incluirse la hipotética resurrección de la reina Isabel I o el ejemplo de Juana de Arco considerado en la Historia de Inglaterra- $)^{21}$.

Pero, se dirá, ¿no es esto igualmente dogmático? ¿No resulta dogmático admitir la posibilidad de lo extraordinario (y la credibilidad de su testimonio bajo ciertas muy exigentes condiciones) y negar la posibilidad de lo milagroso (y la credibilidad de su testimonio bajo cualquier circunstancia)? Y la respuesta es que lejos de resultar dogmático lo que resulta es escéptico. Pues lo que Hume estaría defendiendo es que absolutamente ninguna evidencia nos obliga a hacer una interpretación religiosa de un evento, por extraordinario que este sea. Si este acontece, el razonador justo reconocerá su ignorancia acerca de cómo puede darse una explicación del mismo conforme a las leyes naturales que conocemos... pero confiará en que esta podría encontrarse. O dicho de otra manera, confiará en que lo que parece contradecir las leyes naturales

${ }^{20}$ Véase D. Hume, The History of England: From the Invasion of Julius Caesar to the Revolution in 1688. 6 vols. Indianapolis, Liberty Classics, 1983-1985. Vol. II. pp. 398-404.

${ }^{21}$ R.G. Meyers hizo pivotar su defensa de Hume sobre esta distinción entre lo maravilloso y lo milagroso en su aportación a la 20th. Hume Conference celebrada en Ottawa en 1994: "The Marvelous and Miraculous: A Defense of Hume", texto que está disponible on line en la dirección: file://Users/ macbookair/Desktop/todo/Hume/Miracles/The\%20Marvelous\%20and\%20Miraculous:\%20A\%20 Defense $\% 20$ of\%20Hume.webarchive

No obstante, nuestra vindicación de Hume discurre por derroteros diferentes, aunque no incompatibles, a la suya.

Araucaria. Revista Iberoamericana de Filosofia, Política, Humanidades y Relaciones Internacionales, año 20, ${ }^{\circ} 40$. Segundo semestre de 2018. Pp. 269-283. ISSN 1575-6823 e-ISSN 2340-2199 doi: 10.12795/araucaria.2018.i40.12 
simplemente no es conforme con ellas -que somos, en definitiva, frente a lo extraordinario, como el príncipe indio para quien resultaba increíble la existencia del hielo dado que no había tenido experiencia del mismo ${ }^{22}$.

Así, pues, quien da un significado religioso a un evento extraordinario no lo hace movido por la evidencia sino... por su fe. Tenemos aquí la exacta contrapartida de la conclusión de Filón/Hume en el ámbito de la religión natural. Si decíamos que la constatación del orden natural solo puede convertirse en evidencia teísta o deísta si quien la realiza ya está religiosamente predispuesto, podemos decir ahora que la constatación de un evento extraordinario, que no se ajusta al orden natural que conocemos, solo puede convertirse en evidencia teísta o deísta si quien lo constata ya está religiosamente predispuesto. No son los milagros los que justifican la fe, sino la fe la que explica que creamos en los milagros.

Una última observación. Si no nos equivocamos toda la discusión de la posición de Hume en términos de la teoría Bayesiana de la probabilidad es ociosa $^{23}$, pues para Hume la probabilidad que correspondería a un evento que se pretendiera milagroso en un sentido estricto o religioso sería siempre, desde la óptica de quien decide mantenerse en la perspectiva naturalista, 0 .

Quien considera que el argumento de Hume es un "abject failure", termina tratando la creencia religiosa como si de una creencia científica se tratara. Si es coherente, terminará suscribiendo la reivindicación de que junto con la teoría de la evolución se expliquen en las clases de biología las del diseño inteligente. La inmensa superioridad filosófica de Hume sobre estos positivistas simplistas es haber contribuido con su argumento a que vivamos en un tiempo en que esta equiparación de lo religioso y lo científico nos parezca una abyecta estupidez. Por haber contribuido a conformar esta separación de ciencia y religión, propia de las sociedades secularizadas en las que vivimos, es por lo que el argumento de Hume, a pesar de todas las deficiencias de su formulación, es una pieza fundamental de la filosofía moderna.

\footnotetext{
22 "El príncipe indio que rehusó creer las primeras narraciones sobre los efectos de las heladas razonaba correctamente y, naturalmente, se requirió un testimonio muy fuerte para ganar su asentimiento a unos hechos provocados por un estado de la naturaleza con el que no estaba familiarizado, y tan poco análogo a aquellos eventos de los que había tenido una experiencia uniforme y constante. Aunque no fueran contrarios a su experiencia, no se conformaban con ella”. EHU. 10.10.

${ }_{23}$ Discusión muy prolífica, por otra parte. Al lector interesado remitimos al trabajo de D. Owen, "Hume versus Price on Miracles and Prior Probabilities: Testimony and the Bayesian Calculation" en P. Millican (Ed.), Reading Hume on Human Understanding. Oxford, Clarendon Press, 2002.
} 


\section{Referencias bibliográficas:}

T. Beauchamp, "Introduction" to D. Hume, An Enquiry concerning Human Understanding. Oxford, University Press, 2006.

F. Beckwith, David Hume's Argument against the Miracles. A Critical Analysis. New York, University Press of America, 1989.

D. Coleman "Hume, Miracles, and Lotteries". Hume Studies. vol. 14, n. 2 , 1988.

J. Earman, Hume's Abject Failure, Oxford, University Press, 2000.

A. Flew Hume's Philosophy of Belief. London, Routledge \& Kegan Paul, 1961.

R.J. Fogelin, "What Hume Actually Said About Miracles". Hume Studies. vol.16, n.1, 1990.

D. Garrett, "Hume on Testimony concerning Miracles" en P. Millikan (Ed.), Reading Hume on Human Understanding. Oxford, Clarendon Press, 2002.

J.C.A. Gaskin, Hume's Philosophy of Religion_. London, MacMillan Press, 1978.

D. Hume, The History of England: From the Invasion of Julius Caesar to the Revolution in 1688. 6 vols. Indianapolis, Liberty Classics, 1983-1985.

D. Hume, Escritos impíos y antirreligiosos. Madrid, Akal, 2005 (edición de José Luis Tasset ).

D. Hume, Principal Writings on Religion: including Dialogues Concerning Natural Religion; and The Natural History of Religion / David Hume; edited with an introduction by J. C. A. Gaskin, Oxford: Oxford University Press, 2008.

D. Hume, T. Beauchamp, An Enquiry concerning Human Understanding. Clarendon Hume edition series, Oxford, Clarendon Press, 2010.

Galileo Galilei, Cartas copernicanas. Madrid, Ediciones Alhambra, 1986.

M.P. Levine, Hume and the Problem of Miracles: A Solution. Dordrecht, Kluwer Academic Publishers, 1989.

J.L. Mackie, The miracle of theism: Arguments for and against the Existence of God. Oxford, Clarendon Press, 1982.

R.G. Meyers 20th. Hume Conference celebrada en Ottawa en 1994: "The Marvelous and Miraculus: A Defense of Hume", Otawa 1994, disponible en file://Users/ macbookair/Desktop/todo/Hume/Miracles/The\%20 Marvelous\%20and\%20Miraculous:\%20A\%20 Defense\%20of\%20Hume. webarchive

P. Millican, "Twenty Questions about Hume's 'Of Miracles" en A. O'Hear (Ed.), Philosophy and Religion. Cambridge University Press, 2011.

J. Ortega y Gasset "Ideas y creencias", en Obras completas, vol. V, Taurus/ Fundación Ortega, Madrid, 2012. 
D. Owen, "Hume versus Price on Miracles and Prior Probabilities: Testimony and the Bayesian Calculation" en P. Millican (Ed.), Reading Hume on Human Understanding. Oxford, Clarendon Press, 2002.

T. Penelhum Hume. New York, St. Martin's Press, 1975.

M. A. Quintanilla, D. Hume, Historia natural de la religión. Diálogos sobre la religión natural. Salamanca, Sígueme, 1974.

V. Sanfélix, Investigación sobre el entendimiento humano. Madrid, Istmo, 2004.

J.H. Sobel, "On the Evidence of Testimony for Miracles: A Bayesian Reconstruction of David Hume's Analysis". Philosophical Quarterly, vol. 37, n. 147, 1987.

R. Swinburne, The concept of Miracle. London, Palgrave MacMillan, 1970.

F. Wilson, "The Logic of Probabilities in Hume's Argument against Miracles". Hume Studies. Vol. 15, n.2, 1989.

L. Wittgenstein, Sobre la certeza. Barcelona, Gedisa, 1998.

K.E. Yandell, Hume's 'Inexplicable Mystery'. His Views on Religion. Philadelphia, Temple Press, 1990. 
